\title{
El aula invertida como estrategia didáctica para la generación de competencias: una revisión sistemática
}

\section{(The Flipped Classroom as a Didactic Strategy to Build Competencies: a Systematic Review)}

\author{
Valeria C. Sandobal Verón \\ Ma. Bianca Marín \\ Teresita H. Barrios \\ Universidad Tecnológica Nacional (Argentina)
}

DOI: https://doi.org/10.5944/ried.24.2.29027

\section{Cómo referenciar este artículo:}

Sandobal Verón, V. C., Marín, M. B., y Barrios, T. H. (2021). El aula invertida como estrategia didáctica para la generación de competencias: una revisión sistemática. RIED. Revista Iberoamericana de Educación a Distancia, 24(2), pp. 285-308. https://doi.org/10.5944/ried.24.2.29027

\section{Resumen}

La clase o aula invertida es una estrategia que comenzó a utilizarse en el nivel superior como una medida para mejorar el desempeño y la motivación de los alumnos. Como una de las primeras actividades del proyecto de investigación en el cual se enmarca el presente artículo, se realizó una búsqueda del estado del arte de la clase invertida en la educación superior. Para esto se realizó una revisión sistemática de la literatura científica, con el objetivo de conocer si ċla metodología de aula invertida se aplica en la educación superior?, ¿cuáles son las herramientas que se utilizan? y si ċlas mismas corresponden a la Web 3.o? Por otro lado, se analizó si la aplicación de esta metodología genera competencias, tales como: aprender en forma continua y autónoma; desempeñarse de manera efectiva en equipos de trabajo; resolver problemas de ingeniería y utilizar de manera efectiva las técnicas y herramientas de aplicación en la ingeniería que se corresponden con las competencias que se buscan en el proyecto en cuestión. El artículo presenta los resultados de la revisión sistemática, de la cual se desprende que el aula invertida se aplica en la educación en ingeniería, que se utilizan diferentes herramientas; pero que esta implementación debe tener ciertas consideraciones para ser aplicada con éxito, como son: la correcta planificación de las actividades a realizar tanto por parte de los alumnos como de los docentes, el conocimiento de ambos actores sobre la metodología a utilizar, entre otras. 
Palabras clave: habilidad; capacidad; ingeniería; estrategia de aprendizaje; universidad.

\begin{abstract}
Flipped classroom is a strategy that began to be used at the higher education as a measure to improve the performance and motivation of students. As one of the first activities of the research project in which this article is framed, a search was made of the state of the art of the flipped classroom in higher education. For this, a systematic review of the scientific literature was carried out, with the aim of knowing if the flipped classroom methodology is applied in higher education, what tools are used and if they correspond to Web 3.0. On the other side, it was analyzed whether the application of this methodology generates competencies, such as: learning continuously and autonomously; perform effectively in work teams; solve engineering problems and effectively use application techniques and tools in engineering; which correspond to the competencies sought in the project under analysis. The article presents the results of the systematic review, which shows that the flipped classroom is applied in engineering education, that different tools are used but that this implementation must have certain considerations to be applied successfully, such as: the correct planning of the activities to be carried out by both the students and the teachers, the knowledge of both actors about the methodology to be used, among others.
\end{abstract}

Keywords: know-how; ability; engineering; learning strategy; university.

Continuamente se busca mejorar el rendimiento académico de los alumnos, que éstos se mantengan motivados para llevar a cabo las actividades propuestas y, recientemente generar competencias que les permitan desenvolverse de manera adecuada en el campo profesional. Para logarlo, se propone incorporar el uso de las TIC como herramientas que propician el buen rendimiento de los alumnos y su involucramiento. Ante estas iniciativas, los roles del alumno y del profesor han ido cambiando, tanto es así, que el alumno pasa a tener una participación más activa y el profesor actúa de tutor más que de expositor.

Una de las propuestas es el modelo que se denomina "Aula invertida" o "Clase invertida" o "Flipped Classroom", donde se utiliza la tecnología multimedia para que los alumnos, antes de la clase, puedan incorporar los temas que serán luego desarrollados de manera más práctica en la clase presencial. Dejando más tiempo así, para las consultas, los intercambios de opiniones y el aprender haciendo.

Respecto al origen del concepto, fueron Jonathan Bergmann y Aaron Sams, dos profesores de química de la Woodland Park High School en Colorado (EEUU), los que consolidaron el término. Actuaron movidos por un objetivo común: conseguir que los alumnos que por diversos motivos no habían podido asistir a clase fueran capaces de seguir el ritmo del curso y no resultaran perjudicados por la falta de asistencia. Para ello decidieron grabar los contenidos docentes a través de un software que permitía capturar en vídeo las presentaciones en Power Point narradas, 
y distribuirlos entre sus alumnos. Sin embargo, poco a poco se dieron cuenta de que las grabaciones no solo las utilizaban aquellos que no habían podido ir a clase, sino la generalidad de sus estudiantes. De este modo comenzaron a invertir su método de enseñanza remitiendo vídeos de las lecciones para que las visualizaran en casa antes de la clase y reservando las horas presenciales para realizar proyectos con los que poner en práctica los conocimientos adquiridos y resolver dudas relacionadas con la materia explicada. Según estos autores -principales dirigentes de la Red de Aprendizaje Flipped-

la Flipped Classroom es un enfoque pedagógico en el que la instrucción directa se mueve desde el espacio de aprendizaje colectivo hacia el espacio de aprendizaje individual, y el espacio resultante se transforma en un ambiente de aprendizaje dinámico e interactivo en el que el educador guía a los estudiantes a medida que se aplican los conceptos y puede participar creativamente en la materia (Berenguer Albaladejo, 2016, p. 1468).

Actualmente, hay investigaciones como la de Rodríguez (2016) cuyo principal objetivo es responder a la pregunta ¿De qué manera se evidencia aprendizaje significativo a partir de la implementación y validación de un modelo Flipped Classroom en un curso de Tecnología Educativa? La investigación busca comprender la perspectiva de los participantes sobre el modelo. Entre los principales resultados se evidenció una percepción positiva de la aplicación del modelo, y la comprensión de las tareas tanto extraclase como interclase; se considera relevante la correcta comunicación, la orientación del docente, el trabajo autónomo y colaborativo. En el artículo publicado por Gunawardena y Liyanage (2018) bajo el título "Flipped classroom using Social Networks: an investigation on learning styles" se investiga sobre los estilos de aprendizaje que los alumnos utilizan en un entorno de aula invertida, utilizando la red social Facebook y la plataforma Moodle que usualmente utilizan.

Actualmente en Argentina, en la enseñanza de la Ingeniería; se está incursionando en la generación de competencias en los alumnos. Podemos definir el concepto de "Competencia" como la capacidad de articular eficazmente un conjunto de esquemas (estructuras mentales)yvalores, permitiendo movilizar(poner a disposición) distintos saberes, en un determinado contexto con el fin de resolver situaciones profesionales. Si se tiene en cuenta que se busca formar a los futuros ingenieros por competencias, que se cuenta con las TIC que están incorporadas al aula, y que la aplicación del aula invertida tiene entre sus objetivos el trabajo autónomo, colaborativo, buscar una actitud analítica y crítica en el alumno; entonces es menester un trabajo que pretenda analizar si el modelo de clase invertida propicia la generación de competencias en los alumnos de Ingeniería en Sistemas de Información.

En este sentido se buscó evidencias al respecto, entre la que se puede destacar, a nivel nacional, la experiencia desarrollada por Ponce et al. (2017), donde se lleva a cabo la implementación de la clase invertida en el curso de Ambientación a la vida 
universitaria -módulo matemática destinados a ingresantes a la Facultad de Ciencias Agropecuarias de la Universidad Nacional de Entre Ríos. Los autores realizan un análisis comparativo de resultados obtenidos con cortes anteriores, donde concluyen que encuentran varias ventajas en la aplicación de este modelo, entre las que se mencionan: la posibilidad de lograr un aprendizaje más profundo, la adquisición de competencias transversales y el aumento de la motivación del estudiante.

Con el objetivo de conocer qué han desarrollado otros autores respecto a la formación de competencias a través del aula invertida y descubrir otras experiencias de implementación sobre aula invertida, se ha realizado una revisión sistemática de la literatura, a través de una metodología confiable y ya probada que se presenta a continuación. Teniendo en cuenta la metodología adoptada, el artículo se estructura de la siguiente manera: en la sección 2 se realiza una descripción de la metodología, en la sección 3 se muestran los resultados obtenidos que dan pie a la sección 4 que permite realizar la discusión de la metodología analizada; y por último la sección 5 donde se describen conclusiones a partir de la contrastación de objetivos planteados y resultados obtenidos.

\section{METODOLOGíA}

La metodología utilizada para realizar el análisis del estado del arte es el denominado proceso de revisión sistemática de la literatura (RSL), basado en la propuesta de Kitchenham y Charters (2007) y, particularmente, la adaptación hecha por Biolchini et al. (2005). Según los autores una revisión sistemática es una metodología para realizar una investigación, que permite recopilar y evaluar la evidencia disponible sobre un tema en particular. A diferencia con el proceso habitual de revisión, en este caso, existen pasos definidos y estrictos que deben respetarse para poder llevar a cabo el proceso. En la figura 1 pueden identificarse las etapas propuestas para desarrollar el proceso de RSL: planificación, ejecución y análisis de resultados. La cuarta etapa resguarda los resultados obtenidos en las etapas de planificación, ejecución y análisis de los resultados; por lo cual se realiza durante todo el proceso. 


\section{Figura 1}

Etapas del proceso RSL

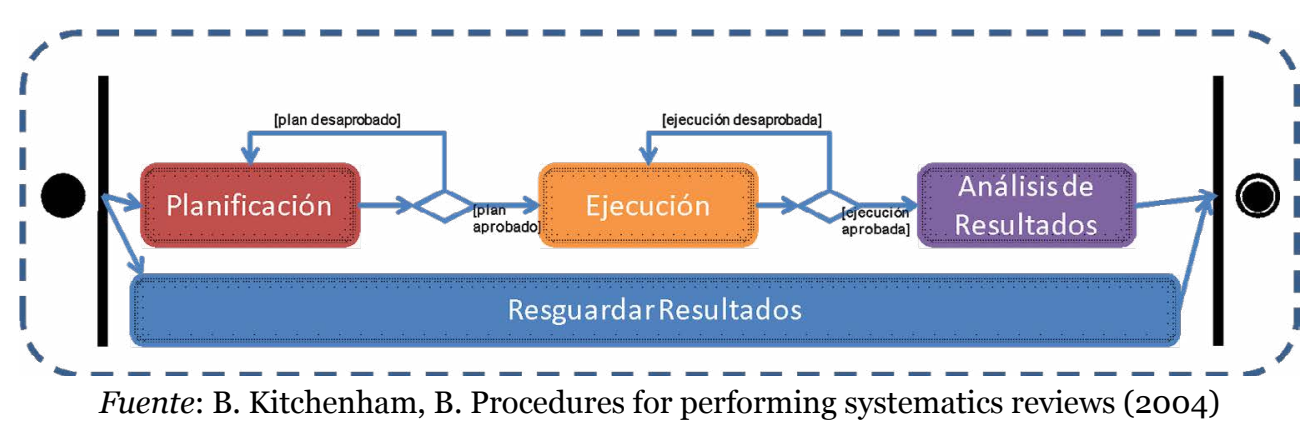

Durante la etapa de planificación se definen los objetivos de la investigación y cómo se llevará a cabo el proceso de revisión; para luego pasar a la ejecución de lo planificado en donde se ejecuta la selección: en una primera instancia de los estudios iniciales, luego la evaluación de calidad y a posteriori se analiza esta última selección. Por último, se deberán analizar los resultados obtenidos, que surgen en una primera instancia por todos los artículos que responden a la cadena de búsqueda sugerida; luego definiendo el corte para el análisis más exhaustivo de los artículos encontrados. En esta etapa, también se seleccionan aquellos artículos que cumplen con los criterios de inclusión y se descartan aquellos según los criterios de exclusión, para luego, evaluar los artículos seleccionados a partir de los criterios de evaluación. Es importante tener en cuenta que todo debe ser documentado, en particular para realizar este trabajo en una primera instancia se indicó de cada artículo encontrado: título, año, fuente de dato, resumen, enlace del artículo y dónde fue publicado. Luego, del primer filtro se resguardó: título, año, fuente de dato y autores.

\section{Preguntas de investigación}

El objetivo definido para esta RSL es el siguiente: "Identificar y analizar el estado del arte de la aplicación de clase invertida en la generación de competencias en la enseñanza de la ingeniería". Luego, a partir del objetivo propuesto se definieron las siguientes preguntas de investigación (PI):

PI1: ¿Se utiliza la metodología de clase invertida en la educación superior y en particular en la formación de ingenieros? Se conoce evidencia de que la clase invertida es una metodología que actualmente se está aplicando a la educación superior, pero en particular se quiere indagar si esta metodología es utilizada en la formación de ingenieros. 
PI2: ¿Las clases invertidas generan competencias en los alumnos? ¿Qué tipo de competencias? En especial interesa conocer si el hecho de invertir la clase permite generar competencias en los alumnos y en el caso de que las generen cuáles son esas competencias. En este sentido, se busca en los artículos, la existencia de evidencias sobre la generación de competencias tales como: aprender de forma continua y autónoma; desempeñarse de manera efectiva en equipo de trabajo; identificar, formular y resolver problemas de ingeniería; y utilizar de manera efectiva las técnicas y herramientas de aplicación en la ingeniería.

PI3: ¿Qué herramientas de la web 3.0 se aplican en la implementación de la clase invertida en la educación superior para generar competencias? Luego de haber determinado que la clase invertida se utiliza en la educación superior para la formación de ingenieros y que generan competencias en los alumnos interesa conocer cuáles son las herramientas de la web 3.0 que permiten llevar a cabo la implementación de la clase invertida.

\section{Fuentes de datos}

La etapa de planificación incluye la selección de las bases de datos en las cuales se realizará la búsqueda de trabajos relacionados. Se seleccionaron las siguientes bases de datos para realizar el estudio de revisión sistemática (se indica nombre de base de datos y URL):

- ACM Digital Library (en adelante ACM): https://dl.acm.org/

- Google Académico: https://scholar.google.com/

- IEEE Xplore Digital Library (en adelante IEEE): https://ieeexplore.ieee.org/ Xplore/home.jsp

- Springer Link (en adelante Springer): https://link.springer.com/

- Science Direct (en adelante SD): https://www.sciencedirect.com/

- Redalyc: https://www.redalyc.org/

$\mathrm{Al}$ definir las cadenas de búsqueda se pretende alcanzar las palabras claves definidas por el objetivo de investigación, las cuales están conformadas por:

- Aula invertida $\rightarrow$ flipped classroom

- Ingeniería $\rightarrow$ engineering

- Competencias $\rightarrow$ soft skills, performance, abilities, self-regulated learning

Luego de varias iteraciones buscando la mejor opción para la cadena de búsqueda se definió la siguiente: 
flipped classroom+engineering + [Publication Date from (01/01/2017]

Las cadenas de búsqueda adaptadas, de acuerdo con la sintaxis de cada fuente de datos se muestra en la tabla 1.

\section{Tabla 1}

Bases de datos con sus respectivas cadenas de búsqueda y alcance

\begin{tabular}{lll} 
Bases de datos & \multicolumn{1}{c}{ Cadena de búsqueda } & Alcance \\
\multirow{2}{*}{ ACM } & [All: “flipped classroom”] AND [All: “soft skills”] AND & \\
& [All: engineering] AND [Publication Date: (o1/o1/2017 TO & Todo \\
& 04/30/2020)] & \\
\hline Google Academic & flipped+classroom+engineering & Todo \\
\hline \multirow{2}{*}{ IEEE } & ((“Abstract”:flipped classroom) AND “Full Text & Abstract \\
\hline SD & Only”:engineer) & Texto \\
\hline \multirow{2}{*}{ Springer } & flipped classroom+engineering & Todo \\
\hline Redalyc & flipped AND classroom AND engineer' & Todo \\
\hline
\end{tabular}

En el caso particular de la fuente de datos Redalyc se incluyen filtros adicionales tales como: educación, psicología, ingeniería, y varios no especificados exactamente; para ajustar la búsqueda a los objetivos propuestos. Para la fuente de datos de Google Academic se filtraron artículos únicamente en español y no se incluyeron citas y patentes con el fin de que la búsqueda arroje resultados acordes a los objetivos de la investigación.

\section{Criterios de inclusión y exclusión}

Al realizar la búsqueda puede haber resultados que no cumplan con las condiciones para ser analizadas y tenidas en cuenta dentro de la RSL, por lo cual se establecieron criterios de inclusión y exclusión. Siguiendo a Kitchenham y Charters (2007), estos criterios se establecen a partir de las preguntas de investigación para poder filtrar los estudios obtenidos.

Con base en lo anterior, se incluyeron aquellos estudios que cumplan con al menos 1 de los siguientes criterios:

- En el título y en las palabras claves identificar palabras relacionadas con el estudio como ser clase invertida (o aula invertida) o ingeniería.

- En el abstract, identificar el objetivo del mismo, y si se realiza una aplicación práctica del aula invertida, es decir, el estudio no solo propone una manera de aplicar aula invertida, sino que muestra los resultados de implementarla. 
- Artículos escritos en inglés o español

Respecto a los criterios de exclusión, se propusieron los siguientes:

- Artículos que no fueran revisiones sistemáticas

- Artículos cuyas investigaciones fueran aplicadas en educación superior de grado

\section{Evaluación de calidad}

Dentro del proceso de revisión sistemática, se realizó la evaluación de calidad de los estudios primarios, de manera tal de poder seleccionar aquellos mejores resultados que luego permitieran interpretar los resultados sin confusiones e interferencias, permitiendo guiar correctamente el proceso de investigación (Carrizo y Moller, 2018).

Con los artículos que quedaron seleccionados, luego de los criterios de inclusión y exclusión, se realizó la verificación de calidad. Los autores Kitchenham y Charters (2007) definieron 6 preguntas de verificación de calidad.

Las preguntas de calidad que se contestaron luego de la lectura de cada uno de los artículos fueron:

- VC1. ¿Cuán claro y coherente es el estudio?

- VC2. ¿Cuán claro es el objetivo del estudio?

- VC3. ¿Qué tan clara es la relación de trazabilidad entre el objetivo del estudio y sus conclusiones?

- VC4. ¿Cuán claro es el proceso de investigación?

- VC5. ¿Qué tan bueno es el estudio comparado con otros relacionados?

- VC6. ¿Cuán claro están definidas las limitaciones del estudio?

Cada una de las preguntas se evalúo con la siguiente puntuación: cumple totalmente $=1$, cumple parcialmente $=0.5 \mathrm{y}$ no cumple $=0$.

\section{Extracción de datos y análisis}

Esta etapa reúne toda la información de los artículos seleccionados que dan respuesta a las preguntas de investigación. En una primera etapa del proceso se agrupó información básica de cada uno de los artículos que permitan identificar a los mismos, como ser:

- Identificación

- Título

- Bases de datos

- Abstract

- Publicado en (conferencia, revista, entre otros) y año de publicación 
Luego, para un mejor entendimiento de cada uno de los artículos se realizó una lectura comprensiva de los mismos para poder identificar: las herramientas que se utilizaron, cómo se llevó a cabo la práctica en el aula, si los resultados obtenidos fueron exitosos, si tuvieron que hacer redefiniciones sobre la propuesta inicial y cuáles se consideran como ventajas y desventajas de la aplicación del flipped-learning.

\section{Proceso que conduce la revisión}

En forma resumida se detalla la secuencia de pasos utilizada para el proceso de revisión:

- $\quad$ Paso $\mathrm{N}^{\circ}$ : Selección de las bases de datos: esto se realizó según el ámbito en donde se enmarcan los estudios y el objetivo buscado.

- Paso $\mathrm{N}^{\circ}$ 2: Selección de las preguntas de investigación: se escogieron aquellas preguntas que respondieron a los objetivos de la investigación y que permitieran guiar el proceso de búsqueda adecuadamente.

- $\quad$ Paso $\mathrm{N}^{\circ} 3:$ Selección de cadena de búsquedas: se propuso inicialmente un conjunto de palabras claves que se consideraron relevantes para realizar las búsquedas. $\mathrm{Al}$ realizar búsquedas de prueba se obtuvieron resultados no deseados, por esto se fueron refinando las cadenas de búsquedas hasta seleccionar la que arrojaba valores más representativos.

- $\quad$ Paso No 4 : Resultados por aplicación de cadena de búsqueda: se aplicó la cadena de búsqueda seleccionada en el paso $\mathrm{N}^{\circ} 3$ a las bases de datos seleccionadas en el paso $\mathrm{N}^{\circ} 1$ y se obtuvieron los primeros resultados.

- Paso $\mathrm{N}^{\circ} 5$ : Selección de criterios de inclusión y exclusión: se definieron los criterios de inclusión y exclusión que permitieran obtener los artículos más adecuados para realizar el análisis final.

- $\quad$ Paso $\mathrm{N}^{\circ} 6$ : Resultados por criterios de exclusión e inclusión: se emplearon los criterios de inclusión y exclusión seleccionados en el paso $\mathrm{N}^{\circ} 5$ a los resultados obtenidos en paso $\mathrm{N}^{\circ} 4$, mediante la lectura del resumen y las conclusiones de cada artículo y se obtuvieron aquellos que se encuentran más relacionados con los objetivos propuestos. En la tabla 2 "Resumen de artículos encontrados, analizados, relevantes y primarios por base de datos", se los puede visualizar en la columna "Relevantes".

- Paso $\mathrm{N}^{\circ} \mathrm{7}$ : Resultados del análisis completo de artículos: nuevamente se aplicaron los criterios de inclusión y exclusión, a los resultados obtenidos en el Paso $\mathrm{N}^{\circ} 6$, pero realizando una lectura completa de cada uno de los artículos, obteniéndose nuevos resultados que son los que figuran en la tabla 2, en la columna "Primarios".

- Paso No8: Resultados por criterios de calidad: se utilizaron los criterios de calidad a los resultados obtenidos en el paso $\mathrm{N}^{\circ} 7$. De la evaluación de calidad de los artículos, se obtuvieron nuevos resultados, aplicándose las preguntas de 
investigación definidas en el punto $\mathrm{N}^{\circ} 2$, a aquellos que pasaron el porcentaje mínimo definido por el equipo (75\%).

- Paso $\mathrm{N}^{\circ}$ : : Resultados Finales: se contestaron las preguntas de investigación definidas en el punto $\mathrm{N}^{\circ}$, con los artículos que pasaron el porcentaje mínimo de calidad definido, los cuales permitirán conducir las propuestas a realizar en el marco del proyecto de aula invertida.

\section{RESULTADOS}

\section{Búsqueda y selección primaria}

Como se mencionó anteriormente, el primer paso fue ejecutar la búsqueda en cada fuente de datos con la correspondiente cadena de búsqueda adaptada a la sintaxis de cada motor. La tabla 2 resume los resultados obtenidos luego de realizar la búsqueda con la cadena definida. Se obtuvieron 17389 resultados.

La segunda columna de la tabla 2 muestra los resultados de cada base de datos; la tercera columna muestra los resultados analizados, donde se consideró en la mayoría de las bases de datos los primeros 50, teniendo en cuenta la cantidad de resultados obtenidos y que los mismos se encuentran filtrados por relevancia. En el caso de Redalyc, se consideraron 72 resultados, ya que fueron incluidas aquellas disciplinas relacionadas con el estudio (educación, psicología, entre otros). En el caso de Science Direct, únicamente 43 resultados, que fueron los que cumplieron las siguientes condiciones: "Flipped Classroom" en título y abstract + "engineering" en texto completo.

En la cuarta columna se indica la cantidad de artículos relevantes, es decir, aquellos que han pasado por los criterios de inclusión y exclusión. Este proceso se realizó teniendo en cuenta título y abstract de los artículos. Se seleccionaron solo 118 artículos, de estos últimos se encontraron 2 repetidos, por lo cual el total a analizar con lectura completa es de 116. Luego, al realizar la lectura completa de los artículos se puede aplicar nuevamente criterios de exclusión, por lo cual la cantidad de estudios primarios es de 95 como se muestra en la columna cinco.

\section{Tabla 2}

Resumen de artículos encontrados, analizados, relevantes y primarios por base de datos

\begin{tabular}{lcccc}
\hline BD & Encontrados & Analizados & Relevantes & Primarios \\
\hline ACM & 152 & 50 & 12 & 9 \\
\hline Google Academic & 16400 & 50 & 15 & 13 \\
\hline IEEE & 108 & 50 & 26 & 21 \\
\hline Springer & 346 & 50 & 21 & 19 \\
\hline
\end{tabular}




\begin{tabular}{lcccc}
\multicolumn{1}{c}{ BD } & Encontrados & Analizados & Relevantes & Primarios \\
\hline Science Direct & 281 & 43 & 22 & 13 \\
\hline Redalyc & 102 & 72 & 22 & 20 \\
\hline TOTALES & $\mathbf{1 7 3 8 9}$ & $\mathbf{3 1 5}$ & $\mathbf{1 1 8}$ & $\mathbf{9 5}$ \\
\hline
\end{tabular}

Ahora bien, si se tuviera que analizar la relevancia de cada fuente de dato, se podría afirmar que Redalyc y la IEEE han tenido porcentajes de relevancia similares entre un $21 \%$ y $24 \%$. La figura 2 muestra las relaciones entre la cantidad de estudios encontrados y relevantes, donde se puede notar una clara relevancia de las dos fuentes antes mencionadas. Además, queda en evidencia el porcentaje de artículos relevantes (en la figura mostrada en naranja) sobre la enorme cantidad de artículos encontrados según las cadenas de búsquedas seleccionadas.

\section{Figura 2}

Relación entre artículos encontrados y relevantes por Base de Dato

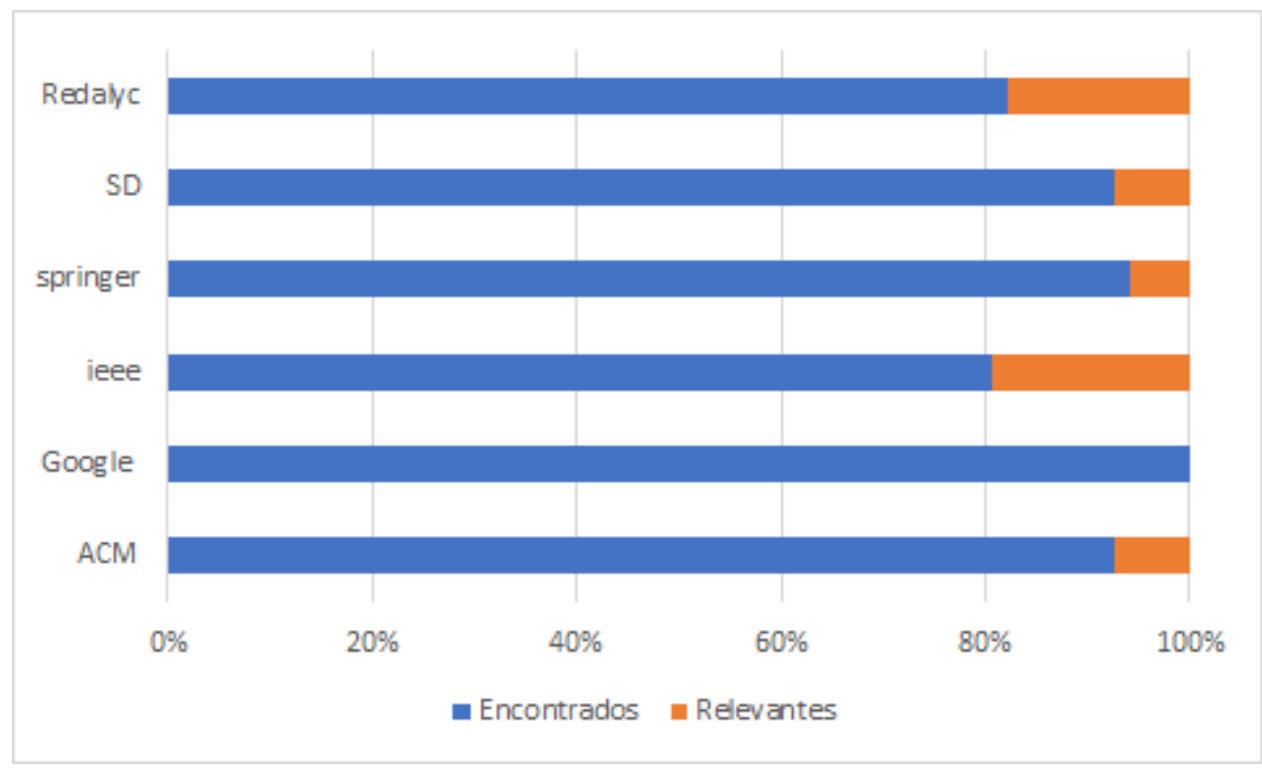

\section{Resultados de la evaluación de calidad}

Con los artículos seleccionados en la etapa anterior, se realizó la evaluación de la calidad de estos, con las preguntas definidas en la sección 2.4.

Al realizar la evaluación de calidad de los 95 artículos, solo 37 fueron los que obtuvieron un porcentaje mayor o igual al $75 \%$. Estos artículos son los que 
permitieron responder a las preguntas de investigación definidas en la sección 2.1. En la tabla 3 se muestran los artículos finalmente seleccionados.

\section{Tabla 3}

\section{Artículos seleccionados}

\section{Título}

Impacts of a flipped classroom with a smart learning diagnosis system on students' learning performance, perception, and problem solving ability in a software engineering course. (Lin, Y. T.)

On the relationships between behaviors and achievement in technology-mediated flipped classrooms: A two-phase online behavioral PLS-SEM model (Wang, F. H.)

A successful experience with the flipped classroom in the Transport Phenomena course. (Valero, M.M., Martínez, M., Pozo, F. y Planas E.)

Perception and performance in a flipped Financial Mathematics classroom. (Lopes, A. P. y Soares, F.)

The role of self-regulated learning in students' success in flipped undergraduate math courses. (Sun, Z., Xie, K. y Anderman, L. H.)

Effectiveness of flip teaching on engineering students' performance in the physics lab. (Gómez-Tejedor, J. A., Vidaurre, A., Tort-Ausina, I., Molina-Mateo, J., Serrano, M. A., Meseguer-Dueñas, J. M., Martínez Sala, R.M., Quiles, S. y Riera, J.)

Micro flip teaching $\square$ An innovative model to promote the active involvement of students. (Fidalgo-Blanco, A., Martínez-Nuñez, M., Borrás-Gene, O. y Sánchez-

Medina, J. J.)

Impact of partially flipped instruction on immediate and subsequent course performance in a large undergraduate chemistry course. (He, W., Holton, A. J. y 2018 Farkas, G.)

Perspectiva estudiantil del modelo pedagógico flipped classroom o aula invertida en el aprendizaje del inglés como lengua extranjera. (Aguayo Vergara, M., Bravo Molina, M., Nocetti de la Barra, A., Concha Sarabia L. y Aburto Godoy, R.)

Clase invertida para el desarrollo de la competencia: uso de la tecnología en estudiantes de preparatoria. (Flores, L.G., Veytia Bucheli, M. G y Moreno Tapia, J.)

El aula presencial, semipresencial, virtual e invertida: Un estudio comparativo de métodos didácticos en la enseñanza de L2. (Bachelor, J. W.)

Experiencia del aula invertida para promover estudiantes prosumidores del nivel superior. (Fernández, M. O. G. y Gaytan, P. H.)

La clase invertida en la educación superior: percepciones del alumnado. (Vera, G. A., Lledó, A. L., Puerta, M. G. y Lledó G.L.) Implementación de aula invertida en un curso propedéutico de habilidad matemática en bachillerato. (Madrid García, E. M., Angulo Armenta, J., Prieto Méndez, M. E., Fernández Nistal, M. T., y Olivares Carmona, K. M.) 
Aprendizaje invertido para la mejora y logro de metas de aprendizaje en el Curso de Metodología de la Investigación en estudiantes de universidad. (MatzumuraKasano, J. P., Gutiérrez-Crespo, H., Zamudio-Eslava, L. A. y Zavala-Gonzales, J. C.)

Integración de TIC de la Web 2.o en el campus virtual universitario de la UCLM. (Estudio inter-sujetos). (Ruiz, A. P., Martínez, A. C. y Perales, R. G.)

Estudio de caso del modelo clase invertida en un curso de mecánica de cuerpos rígidos. (Ramírez, Y. G.)

Effect of Instructional Methods on Student Performance in Flipped Classroom. (Nwokeji, J. C., Stachel, R. y Holmes, T.)

Analyses of possibilities of Flipped Classroom in Teaching Computer Science Courses. (Fetaji, M., Fetaji, B. y Ebibi, M.)

Improving student learning in an introductory programming course using flipped classroom and competency framework. (Elmaleh, J., y Shankararaman, V.)

The Self-Flipped Classroom Concept: Underlying Ideas and Experiences.

(Vasilchenko, A., Cajander, Å., Daniels, M. y Balaam, M.)

2018

Impact of Flipped Physics Classes on the Performance of Engineering Students: University of Sharjah Case Study. (Elmehdi, H. M., Dalah, E. Z. e Ibrahem, A. M.)

Classroom partial flip for feedback control systems: A biomedical engineering experience. (de Moura Oliveira, P. B. y Cunha, J. B.)

Supporting Intensive Continuous Assessment With BeA in a Flipped Classroom Experience. (Llamas-Nistal, M., Mikic-Fonte, F. A., Caeiro-Rodríguez, M. y Liz-

Domínguez, M.)

Learning gain study in a strategy of flipped learning in the undergraduate level. (Juárez, G. H. y Herrera, L. M. M.)

Investigating Flipped Learning: Student Self-Regulated Learning, Perceptions, and Achievement in an Introductory Biology Course. (Sletten, S. R.)

Using Open-Source Videos to Flip a First-Year College Physics Class. (Robinson, F. J., Reeves, P. M., Caines, H. L. y De Grandi, C.)

Research on blended teaching model in the teaching of 'micro-controller principles and applications'. (Tong, Y. y Song, P.)

Analytics of Learning Strategies: Associations with Academic Performance and Feedback. (Matcha, W., Gašević, D., Uzir, N. A. A., Jovanović, J. y Pardo, A.)

Implementación de flipped classroom enfocado a los estudiantes de Ingeniería de software: caso universidad ecuatoriana. (Álvarez, D. M. L., Aguilar, G. F. C., Conforme, N. C. R. y Alcívar, I. A. M.)

Flipped classroom como estrategia metodológica para mejorar la competencia en trabajo grupal en didáctica de la matemática. (Sánchez-Cruzado, C., SánchezCompaña, T. y Macías-García, J. A.) 


\section{Título}

Desarrollo de competencias genéricas mediante flipped classroom y el uso de herramientas web 2.0 en el marco de trabajos colaborativos en educación superior. 2017 (Biel, P., Pérez Sinusía, E., Serrano, A., y Rodrigo, C.)

Flipped classroom y el efecto en las competencias transversales de los alumnos del curso de electricidad y electrónica industrial en una universidad pública de Lima.

2018 (Benites Yarleque, J. V.)

Método para Promover el Aprendizaje Colaborativo en Ingeniería de Software. (Cornide-Reyes, H. C. y Villarroel, R. H.) 2019 Ecuaciones diferenciales con aula invertida. (Jambrina, L. F.) 2017

Aprendizaje invertido con Elevator Pitch y Pecha Kucha. (Romero, E., Artal, J. S., Artacho, J. M. y García Aranda, J. R.)

\section{DISCUSIÓN}

En esta sección se responderá a las preguntas de investigación planteadas en la sección 2.1, para ello se usarán los artículos seleccionados en la sección anterior.

PI.1 ¿Se utiliza la metodología de clase invertida en la educación superior o en la formación de ingenieros?

En una primera instancia se busca que la clase invertida se haya aplicado a la educación superior. Sin embargo, dentro de esta muestra interesa conocer aquellas experiencias que fueron aplicadas directamente a la formación de ingenieros, es así como en 18 artículos se encuentran experiencias que han aplicado la metodología de aula invertida en carreras de ingeniería. En la figura 3 se puede visualizar la relación entre el total de artículos que han pasado los criterios de calidad, aquellos que se enfocan en la aplicación en carreras de Ingeniería y cuáles fueron las bases de datos en las que estos artículos fueron encontrados. 


\section{Figura 3}

Relación entre artículos totales, artículos aplicados a Ingeniería y bases de datos
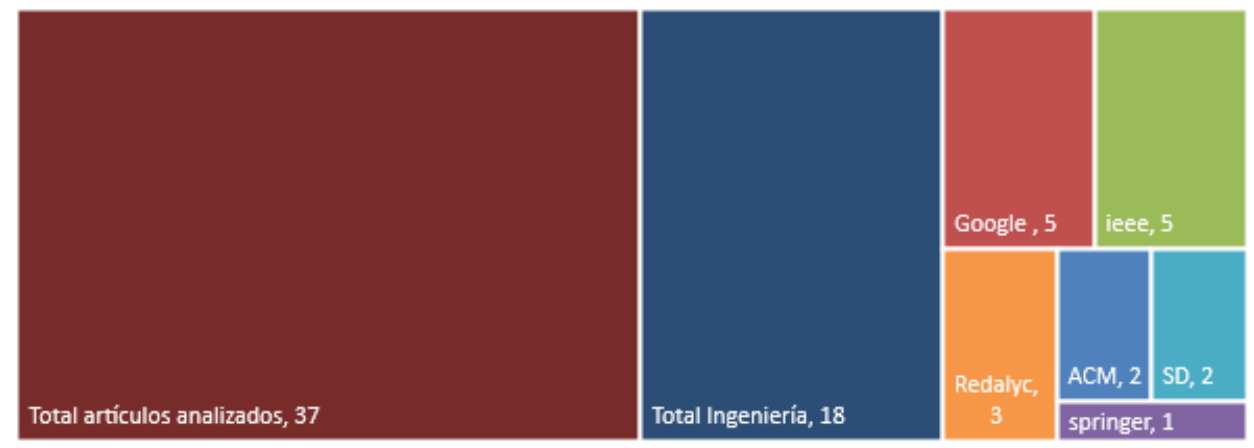

Si se profundiza aún más en las experiencias, es posible encontrar 7 que lo aplican directamente a materias que están relacionadas con la carrera Ingeniería en Sistemas de Información y afines.

PI.2 ¿Las clases invertidas generan competencias en los alumnos? ¿Qué tipo de competencias?

En especial interesa conocer si el hecho de invertir la clase permite generar competencias en los alumnos y en el caso de que las generen cuáles son esas competencias. En este sentido, se buscará en la evidencia de los artículos la generación de competencias tales como: aprender de forma continua y autónoma; desempeñarse de manera efectiva en equipo de trabajo; identificar, formular y resolver problemas de ingeniería; y utilizar de manera efectiva las técnicas y herramientas de aplicación en la ingeniería. En cuanto a las competencias seleccionadas, se identificaron por un lado aquellas que son competencias sociales, políticas y actitudinales, que son transversales a la mayoría de las materias para la formación de los alumnos en la carrera que se tomará como muestra para el proyecto de investigación que se está realizando. Luego, por el lado de las competencias tecnológicas se tomaron dos de las que son comunes en las materias que se tomarán de referencia. En la figura 4 se puede visualizar las competencias generadas según los artículos analizados; en donde se observa un mayor porcentaje en cuanto a la búsqueda de motivación. Luego, el aprendizaje autónomo, y en menor medida la resolución de problemas, el trabajo colaborativo y la comunicación efectiva. 


\section{Figura 4}

Competencias encontradas en los artículos analizados

\section{Competencias encontradas}

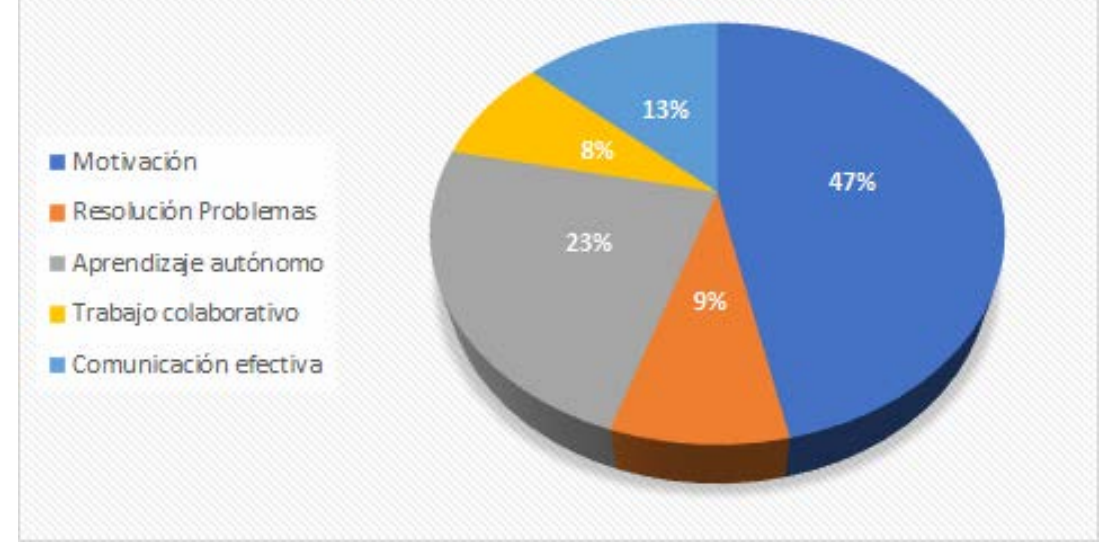

PI.3 ¿Qué herramientas de la web 3.0 se aplican en la implementación de la clase invertida en la educación superior para generar competencias?

Las herramientas más utilizadas para invertir el aula, según los artículos analizados durante el proceso de revisión sistemática, fueron los videos. A su vez se puede mencionar otras herramientas, como ser Prezi, Genially, Moovly o PowToon mencionadas en el artículo Biel et al. (2017), PlayPosit, Kahoot y Socrative mencionadas en el artículo de Álvarez et al. (2020) y blogs, animaciones, podcast, wikis, resúmenes interactivos y juegos digitales mencionados en el artículo de Vasilchenko (2018), entre otras. A su vez se proponen métodos como ser el desarrollado por Cornide-Reyes y Villarroel (2019) que utiliza Byos (Build Your Own Scrum, Crea tu propio Scrum), para la materia de Ingeniería de Software.

Todas estas herramientas e implementaciones resultan muy interesantes e innovadoras a la hora de seleccionar una solución adecuada para invertir la clase, que implican un desafío y compromiso para motivar al alumno en la implementación de la metodología. En la mayoría de los casos se menciona la importancia del compromiso docente en la implementación, manifiesto en la información y formación que hace de sus alumnos para trabajar con esta estrategia.

Ahora bien, estas experiencias no nos permiten responder a PI3, ya que lo que se busca es la implementación de herramientas de la web 3.o como ser Facebook, Instagram, Google+, Twitter, entre otras. Se considera que las redes sociales pueden ser una herramienta posible de utilizar en la aplicación de la clase invertida 
considerando que según el estudio realizado por Xie (2019) en Argentina el 78\% de la población es usuario de internet, y de ese porcentaje el $76 \%$ son usuarios de redes sociales. Aún más los jóvenes que se encuentran habituados a la utilización de estas herramientas.

\section{CONCLUSIONES}

La revisión sistemática surge con el objetivo de determinar cuál es el estado del arte en lo referente a la utilización de las aulas invertidas para la formación de competencias en estudiantes universitarios. "Analizar, apropiar, aplicar y contextualizar referentes teóricos y procedimentales sobre estado del arte y revisión sistemática de literatura se constituyen como bases sólidas para la generación de una metodología de RAD” (Chacón et al., 2013, p. 101).

Habiéndose realizado un análisis exhaustivo donde se seleccionaron 6 bases de datos y cadenas de búsqueda adaptadas a cada una de ellas, y habiéndose definido criterios de inclusión y exclusión, se pudieron seleccionar estudios que luego fueron evaluados según criterios de calidad, que al finalizar dieron como resultado 37 artículos. Esto nos permite visualizar que la temática se encuentra en estudio y crecimiento; haciendo una salvedad: pocos de estos estudios fueron aplicados a carreras de ingeniería y menos aún de Ingeniería en sistemas de información o afines.

Las competencias mayormente referenciadas en los distintos artículos se pueden destacar: comunicación, autorregulación, trabajo en equipo, creatividad, entre otras; todas ellas competencias deseables de desarrollar en un estudiante universitario, que fueron incluidas en el proyecto de investigación en curso.

La mayoría de los artículos mencionan la motivación de los alumnos, que si bien, no es una competencia explícitamente indicada en el trabajo de investigación resulta de vital importancia porque al aplicar la clase invertida se pretende un cambio de paradigma, sobre todo en cuanto al tipo de participación de los actores involucrados. En 3 casos de aplicación de la clase invertida en Ingeniería en Sistemas de Información o carrera afines se menciona como positiva esta competencia. Se destaca el caso de Tong y Song (2018) donde se implementa blended-learning combinando el aprendizaje basado en proyectos, aprendizaje colaborativo y aula invertida en un curso para comprender y aplicar microcontroladores, donde la preclase está más relacionada con la comprensión de conceptos teóricos, para luego durante la clase desarrollar la implementación de esos conceptos comprendidos.

Luego, la experiencia presentada por Álvarez et al. (2020) desarrollada en la asignatura Ingeniería de Software que, según los mismos autores, generalmente es una materia que presenta poco interés por parte de los alumnos por la carga teórica de la misma. Al realizar la experiencia no solo pudieron notar un aumento en la motivación de los alumnos, sino también un notable mejoramiento del rendimiento académico. 
Por último, la implementación de aula invertida combinada con Scrum en la carrera de Ingeniería de Software desarrollada por Cornide-Reyes y Villarroel (2019), menciona los resultados alentadores en la motivación de los alumnos, tanto para la comprensión de los contenidos como para el desarrollo de las actividades propuestas.

Elaprendizajedeforma continuayautónoma(aprendizajeautónomooaprendizaje autorregulado o aprendizaje autogestionado y autoaprendizaje) se destaca como una competencia alcanzada satisfactoriamente en 14 de los estudios analizados. Pueden mencionarse estudios donde se enfatiza la responsabilidad asumida por los estudiantes en el proceso de aprendizaje, tal es la experiencia presentada por Matzumura-Kasano et al. (2018, p. 14) quienes entre sus conclusiones afirman que el "estudiantado asume la responsabilidad de construir, entender el conocimiento, reflexionan para crear significado y participar activamente ...”. Asimismo, en Ramírez (2019) se manifiesta que los estudiantes fueron más conscientes de sus propios aprendizajes. En este mismo sentido, en Lopes y Soares (2018) se observa que los alumnos asumen la responsabilidad de sus propios aprendizajes y ven reflejados esos aprendizajes en los resultados obtenidos.

Desempeñarse de manera efectiva en equipos o trabajo colaborativo, se encuentran explícitamente mencionados como competencia generada y con resultados positivos luego de la implementación del aula invertida, en 6 de los casos. Si se toman los que se aplicaron en carreras de Ingeniería en sistemas de información o afines, se destacan 2 trabajos: en Nwokeji et al. (2019) se menciona como un factor importante al momento de realizar proyectos. También, Cornide-Reyes y Villarroel (2019) mencionan el trabajo en equipo de vital importancia, teniendo en cuenta que se mezcla la metodología de aula invertida con la práctica ágil Scrum. En esta última se tiene como base el trabajo en equipo buscando como principales características los equipos autogestionados y multifuncionales.

Si se toma como base, que la implementación del aula invertida tiene como una de sus principales actividades durante la clase la aplicación práctica de los temas de las materias; es de suponer que se podría llegar a generar la competencia de resolución de conflictos en los alumnos. Sin embargo, de manera explícita esta competencia es mencionada en 5 artículos. Lin (2019) se refiere a que el sistema propuesto beneficia a los estudiantes con respecto a la capacidad para la resolución de problemas. También, en Matzumura-Kasano et al. (2018) se la presenta como una posibilidad de participación activa de los estudiantes que los lleva a resolver de manera adecuada las prácticas propuestas. En el caso de Wang (2019), sugiere que la resolución de problemas en clase actúa como motivador para la conducta de aprendizaje de los alumnos fuera de clase, y como para consolidar lo aprendido luego de la clase.

Si bien, la competencia de comunicarse de manera efectiva no fue incluida como una de las competencias seleccionadas para el desarrollo del proyecto de investigación; surge durante el análisis, que 8 de los estudios analizados la 
mencionan. En la experiencia desarrollada por Ruiz et al. (2018, p. 112), se expresa que la aplicación del aula invertida permite a los alumnos realizar "un trabajo más visual, intuitivo, colaborativo y con mayor compromiso e implicación, facilitando una verdadera comunicación multidireccional entre los alumnos". Para Biel et al. (2017), la competencia de comunicación oral se genera a través de la utilización de herramientas de la Web 2.o. Según la percepción de los alumnos, impactó positivamente en esta competencia el uso de dichas herramientas al momento de realizar la presentación de resultados de sus aprendizajes. Por último, Juárez y Herrera (2019), según la experiencia realizada, explican que la aplicación del aula invertida tiene efectos positivos en los alumnos al responsabilizarlos de sus progresos y la posibilidad que este compromiso los lleva a mejorar la forma de interactuar y comunicarse con sus pares y profesores.

Si bien no se considera en el proyecto de investigación la generación de competencias digitales, la misma es mencionada explícitamente en 4 de los estudios analizados (Juárez y Herrera, 2019; Álvarez et al., 2020; Sánchez-Cruzado et al., 2018; Biel et al., 2017), entendiéndose como competencia digital, la posibilidad que los alumnos tienen de utilizar las nuevas tecnologías para realizar de manera autónoma sus actividades. En este sentido, se puede considerar de vital importancia para obtener resultados positivos que los alumnos conozcan las herramientas con las que deberán trabajar para que realmente constituya una ventaja y no una desventaja usar las mismas.

En cuanto a las herramientas de la web 3.0 no son utilizadas en los estudios analizados, sin embargo, las herramientas empleadas son diversas. En su mayoría coinciden en el uso de videos y otras utilizadas para su creación y edición, así como campus virtual. A su vez, el estudio realizado permite conocer nuevas variantes, por lo cual se puede concluir que se deben seleccionar las herramientas según las características del entorno en donde deban aplicarse.

A partir delos artículos analizados se recomienda que, al momento de implementar el aula invertida es necesario tener en cuenta las siguientes consideraciones:

- Correcta planificación de las actividades a desarrollar por: los alumnos antes, durante y después de clase; por los profesores para diseñar los recursos a utilizar y las actividades a desarrollar.

- Conocimiento de la metodología a usar en clase. Incluida la obligatoriedad o no de las actividades propuestas.

- Contextualizar la propuesta al entorno en el que se va a aplicar, teniendo en cuenta la disponibilidad de recursos para todos los actores.

- Definir rúbricas de evaluación donde se consideren las competencias que serán evaluadas; y cómo serán evaluadas, las cuales deben ser conocidas por los estudiantes antes de comenzar el dictado de la asignatura. 


\section{REFERENCIAS}

Aguayo Vergara, M., Bravo Molina, M., Nocetti de la Barra, A., Concha Sarabia, L., y Aburto Godoy, R. (2019). Perspectiva estudiantil del modelo pedagógico flipped classroom o aula invertida en el aprendizaje del inglés como lengua extranjera. Revista Educación, 43(1), 97-113. https://doi.org/10.15517/revedu. v43i1.31529

Álvarez, D. M. L., Aguilar, G. F. C., Conforme, N. C. R., y Alcívar, I. A. M. (2020). Implementación de flipped classroom enfocado a los estudiantes de Ingeniería de software: caso universidad ecuatoriana. Revista Científica Ecociencia, 7(3), 1-18. https://doi.org/10.21855/ ecociencia.73.311

Bachelor, J. W. (2019). El aula presencial, semipresencial, virtual e invertida: Un estudio comparativo de métodos didácticos en la enseñanza de L2. Revista Educación, 43(2), 541-553. https://doi. org/10.15517/revedu.v43i2.34014

Benites Yarleque, J. V. (2018). Flipped classroom y el efecto en las competencias transversales de los alumnos del curso de electricidad y electrónica industrial en una universidad pública de Lima.

Berenguer Albaladejo, C. (2016). Acerca de la utilidad del aula invertida o flipped classroom. XIV Jornadas de Redes de Investigación en Docencia Universitaria. Universidad de Alicante.

Biel, P., Pérez Sinusía, E., Serrano, A., y Rodrigo, C. (2017). Desarrollo de competencias genéricas mediante flipped classroom y el uso de herramientas web 2.o en el marco de trabajos colaborativos en educación superior [Development of generic competencies through flipped classroom and the use of web 2.0 tools within the framework of collaborative work in higher education] (No. COMPON-2017-0024).
Biolchini, J., Mian, P. G., Natali, A. C. C., y Travassos, G. H. (2005). Systematic review in software engineering. System Engineering and Computer Science Department COPPE/UFRJ. Technical Report ES, 679(05), 45.

Carrizo, D., y Moller, C. (2018). Estructuras metodológicas de revisiones sistemáticas de literatura en Ingeniería de Software: un estudio de mapeo sistemático. Ingeniare. Revista chilena de ingeniería, 26, 4554. $\quad$ https://doi.org/10.4067/So71833052018000500045

Chacón, J. W. B., Herrera, J. C. B., y Villabona, M. R. (2013). Revisión y análisis documental para estado del arte: una propuesta metodológica desde el contexto de la sistematización de experiencias educativas. Investigación Bibliotecológica: archivonomía, bibliotecología e información, 27(61), 83-105. https://doi.org/10.1016/So187358X(13)72555-3

Cornide-Reyes, H. C., y Villarroel, R. H. (2019). Método para Promover el Aprendizaje Colaborativo en Ingeniería de Software. Formación universitaria, 12(4), 3-12. https://doi.org/10.4067/ S0718-50062019000400003

de Moura Oliveira, P. B., y Cunha, J. B. (2017, July). Classroom partial flip for feedback control systems: A biomedical engineering experience. En 2017 25th Mediterranean Conference on Control and Automation (MED) (pp. 957-961). IEEE. https://doi. org/10.1109/MED.2017.7984242

Elmaleh, J., y Shankararaman, V. (2017, April). Improving student learning in an introductory programming course using flipped classroom and competency framework. En 2017 IEEE Global Engineering Education Conference (EDUCON) (pp. 49-55). IEEE. https:// doi.org/10.1109/EDUCON.2017.7942823 
Elmehdi, H. M., Dalah, E. Z., e Ibrahem, A. M. (2019, March). Impact of Flipped Physics Classes on the Performance of Engineering Students: University of Sharjah Case Study. En 2019 Advances in Science and Engineering Technology International Conferences (ASET) (pp. 1-6). IEEE. https://doi.org/10.1109/ ICASET.2019.8714477

Fernández, M. O. G., y Gaytan, P. H. (2019). Experiencia del aula invertida para promover estudiantes prosumidores del nivel superior. RIED. Revista Iberoamericana de Educación a Distancia, 22(2), 245-263. https://doi. org/10.5944/ried.22.2.23065

Fetaji, M., Fetaji, B., y Ebibi, M. (2019, May). Analyses of possibilities of Flipped Classroom in Teaching Computer Science Courses. En 2019 42nd International Convention on Information and Communication Technology, Electronics and Microelectronics (MIPRO) (pp. 747752). IEEE. https://doi.org/10.23919/ MIPRO.2019.8757126

Fidalgo-Blanco, A., Martínez-Nuñez, M., Borrás-Gene, O., y Sánchez-Medina, J. J. (2017). Micro flip teaching - An innovative model to promote the active involvement of students. Computers in Human Behavior, 72, 713-723. https:// doi.org/10.1016/j.chb.2016.07.060

Flores, L. G., Veytia Bucheli, M. G., y Moreno Tapia, J. (2020). Clase invertida para el desarrollo de la competencia: uso de la tecnología en estudiantes de preparatoria. Revista Educación, 44(1), 192-209. https://doi.org/10.15517/revedu. v44i1.36961

Garcia, S. (2018). Improving classroom preparedness using guided practice. En Proceedings of the 49th ACM Technical Symposium on Computer Science Education (pp. 326-331). https://doi. org/10.1145/3159450.3159571

Gómez-Tejedor, J. A., Vidaurre, A., TortAusina, I., Molina-Mateo, J., Serrano,
M. A., Meseguer-Dueñas, J. M., Martínez Sala, R.M., Quiles, S., y Riera, J. (2020). Effectiveness of flip teaching on engineering students' performance in the physics lab. Computers \& Education, 144, 103708. https://doi.org/10.1016/j. compedu.2019.103708

Gunawardena, L., y Liyanage, M. P. P. (2018, July). Flipped Classrooms using Social Networks: An investigation on learning styles. En 20187 th International Congress on Advanced Applied Informatics (IIAIAAI) (pp. 956-957). IEEE. https://doi. org/10.1109/IIAI-AAI.2018.00199

He, W., Holton, A. J., y Farkas, G. (2018). Impact of partially flipped instruction on immediate and subsequent course performance in a large undergraduate chemistry course. Computers \& Education, 125, 120-131. https://doi. org/10.1016/j.compedu.2018.05.020

Jambrina, L. F. (2017). Ecuaciones diferenciales con aula invertida. IV Congreso Internacional sobre aprendizaje, innovacióny competitividad.

Juárez, G. H., y Herrera, L. M. M. (2019). Learning gain study in a strategy of flipped learning in the undergraduate level. International Journal on Interactive Design and Manufacturing (IJIDeM), 13(4), 1245-1258. https://doi. org/10.1007/s12008-019-00594-3

Kitchenham, B., y Charters, S. (2007). Guidelines for performing systematic literature reviews in software engineering.

Lin, Y. T. (2019). Impacts of a flipped classroom with a smart learning diagnosis system on students' learning performance, perception, and problem solving ability in a software engineering course. Computers in Human Behavior, 95, 187-196. https:// doi.org/10.1016/j.chb.2018.11.036

Llamas-Nistal, M., Mikic-Fonte, F. A., Caeiro-Rodríguez, M., y Liz-Domínguez, M. (2019). Supporting Intensive Continuous Assessment With BeA in a 
Flipped Classroom Experience. IEEE Access, 7, 150022-150036. https://doi. org/10.1109/ACCESS.2019.2946908

Lopes, A. P., y Soares, F. (2018). Perception and performance in a flipped Financial Mathematics classroom. The International Journal of Management Education, 16(1), 105-113. https://doi. org/10.1016/j.ijme.2018.01.001

Madrid García, E. M., Angulo Armenta, J., Prieto Méndez, M. E., Fernández Nistal, M. T., y Olivares Carmona, K. M. (2018). Implementación de aula invertida en un curso propedéutico de habilidad matemática en bachillerato. Apertura, 1O(1), 24-39. https://doi.org/10.32870/ Ap.v10n1.1149

Matcha, W., Gašević, D., Uzir, N. A. A., Jovanović, J., y Pardo, A. (2019, March). Analytics of learning strategies: Associations with academic performance and feedback. En Proceedings of the 9th International Conference on Learning Analytics \& Knowledge (pp. 461-470). https://doi. org/10.1145/3303772.3303787

Matzumura-Kasano, J. P., Gutiérrez-Crespo, H., Zamudio-Eslava, L. A., y ZavalaGonzales, J. C. (2018). Aprendizaje invertido para la mejora y logro de metas de aprendizaje en el Curso de Metodología de la Investigación en estudiantes de universidad. Revista Electrónica Educare, 22(3), 177-197.

Nwokeji, J. C., Stachel, R., y Holmes, T. (2019, October). Effect of Instructional Methods on Student Performance in Flipped Classroom. En 2019 IEEE Frontiers in Education Conference (FIE) (pp. 1-9). IEEE. https://doi.org/10.1109/ FIE43999.2019.9028670

Ponce, S. L., Marichal, A., Martínez, G., Soldini, M., y Ponce, R. D. (2017). Implementación de la clase invertida en el aula universitaria: posibilidades para la obtención de aprendizajes no superficiales. En XII Congreso de
Tecnología en Educación y Educación en Tecnología (TE\&ET, La Matanza 2017).

Ramírez, Y. G. (2019). Estudio de caso del modelo clase invertida en un curso de mecánica de cuerpos rígidos. Gaceta Técnica, 2O(2), 51-65.

Robinson, F. J., Reeves, P. M., Caines, H. L., y De Grandi, C. (2020). Using Open-Source Videos to Flip a First-Year College Physics Class. Journal of Science Education and Technology, 1-11. https:// doi.org/10.1007/s10956-020-09814-y

Rodríguez, W. P. (2016). Estudio de evidencias de aprendizaje significativo en un aula bajo el modelo flipped classroom. Edutec. Revista Electrónica de Tecnología Educativa, 55, a325-a325.

Romero, E., Artal, J. S., Artacho, J. M., y García Aranda, J. R. (2017). Aprendizaje invertido con Elevator Pitch y Pecha Kucha. IV Congreso Internacional sobre Aprendizaje, (No. COMPON-2017-0117).

Ruiz, A. P., Martínez, A. C., y Perales, R. G. (2018). Integración de TIC de la Web 2.0 en el campus virtual universitario de la UCLM. (Estudio intersujetos). REXE-Revista de Estudios y Experiencias en Educación, 2(2), 103113. https://doi.org/10.21703/rexe. Especial3 20181031139

Sánchez-Cruzado, C., Sánchez-Compaña, T., y Macías-García, J. A. (2018). Flipped classroom como estrategia metodológica para mejorar la competencia en trabajo grupal en didáctica de la matemática. Matemáticas, educación y sociedad, 1(3), 31-43.

Sletten, S. R. (2017). Investigating flipped learning: Student self-regulated learning, perceptions, and achievement in an introductory biology course. Journal of Science Education and Technology, 26(3), 347-358. https://doi.org/10.1007/ s10956-016-9683-8

Sun, Z., Xie, K., y Anderman, L. H. (2018). The role of self-regulated learning in students' success in flipped undergraduate 
math courses. The Internet and Higher Education, 36, 41-53. https://doi. org/10.1016/j.iheduc.2017.09.003

Tong, Y., y Song, P. (2018, January). Research on blended teaching model in the teaching of 'micro-controller principles and applications'. En Proceedings of the 6th International Conference on Information and Education Technology (pp. 38-41). https://doi.org/10.1145/3178158.3178185

Valero, M. M., Martínez, M., Pozo, F., y Planas, E. (2019). A successful experience with the flipped classroom in the Transport Phenomena course. Education for Chemical Engineers, 26, 67-79. https:// doi.org/10.1016/j.ece.2018.08.003

Vasilchenko, A., Cajander, Å., Daniels, M., y Balaam, M. (2018, October). The selfflipped classroom concept: Underlying ideas and experiences. En 2018 IEEE Frontiers in Education Conference (FIE) (pp. 1-9). IEEE. https://doi.org/10.1109/ FIE.2018.8658616

Vera, G. A., Lledó, A. L., Puerta, M. G., y Lledó, G. L. (2018). La clase invertida en la educación superior: percepciones del alumnado. Revista INFAD de Psicología. International Journal of Developmental and Educational Psychology, 2(1), 155-162. $\quad$ https://doi.org/10.1706o/ ijodaep.2018.n1.v2.1197

Wang, F. H. (2019). On the relationships between behaviors and achievement in technology-mediated flipped classrooms: A two-phase online behavioral PLSSEM model. Computers \& Education, 142, 103653. https://doi.org/10.1016/j. compedu.2019.103653

Xie, Y. M. (2019). Social Media, Marketing, SEO, Marca Personal. https:// yiminshum.com/digital-social-mediaargentina-2019/

\section{PERFIL ACADÉMICO Y PROFESIONAL DE LOS AUTORES}

Valeria Celeste Sandobal Verón. Ingeniería en Sistemas de Información. Jefe de Trabajos Prácticos del Departamento de Ingeniería en Sistemas de Información en la Facultad Regional Resistencia, Universidad Tecnológica Nacional (FRRe - UTN). Investigadora del Grupo de Investigación Educativa sobre Ingeniería (GIESIN). Responsable del área de Recursos Humanos de la Secretaría de Ciencia y Tecnología.

E-mail: vsandobal@frre.utn.edu.ar

DIRECCIÓN DE LA AUTORA

Departamento de Ingeniería en Sistemas de Información

Facultad Regional Resistencia, Universidad Tecnológica Nacional

Edificio Central, French 414

H3500CHJ, Resistencia - Chaco, Argentina 
María Bianca Marín. Ingeniera en sistemas de información, Especialista en ingeniería gerencial, Abogada. Investigadora del Grupo de Investigación Educativa sobre Ingeniería (GIESIN) de la Universidad Tecnológica Nacional - Facultad Regional Resistencia. Coordinadora del equipo de tutorías de la Universidad Tecnológica Nacional - Facultad Regional Resistencia. Desarrolladora de Software empresa ECOM CHACO S.A. Asesoría Informática y jurídica.

E-mail: mbiancamarin@gmail.com

Teresita Haydeé Barrios. Ingeniera en Sistemas de Información, Auxiliar de 1ra en el departamento de Ingeniería en Sistemas de información de la Universidad Tecnológica Nacional (UTN) - Facultad Regional Resistencia (FRRE). Investigadora del Grupo de Investigación Educativa sobre Ingeniería (GIESIN) de la UTN - FRRe. Jefa del servicio de Informática del Hospital Dr. J. C. Perrando, Chaco - Argentina. E-mail: barriosth@gmail.com

\section{DIRECCIÓN DE LAS AUTORAS}

Grupo de Investigación Educativa sobre Ingeniería (GIESIN)

Facultad Regional Resistencia, Universidad Tecnológica Nacional

Edificio Central, French 414

H350oCHJ, Resistencia - Chaco, Argentina

Fecha de recepción del artículo: 02/12/2020

Fecha de aceptación del artículo: 06/02/2021

Fecha de aprobación para maquetación: 12/03/2021 